\title{
Solid State Conformations of Symmetrical Aromatic Biheterocycles: an X-Ray Crystallographic Investigation
}

\author{
Christopher M. Fitchett, Chris Richardson and Peter J. Steel *
}

Chemistry Department, University of Canterbury, Christchurch, New Zealand

Fax:+64-3-3642110; Tel: +64-3-3642432; E-mail: peter.steel@canterbury.ac.nz

\author{
This submission was created using the RSC Article Template (DO NOT DELETE THIS TEXT) \\ (LINE INCLUDED FOR SPACING ONLY - DO NOT DELETE THIS TEXT)
}

Accurate, low temperature X-ray crystal structure determinations show that 3,3'-biquinoline (6), 2,2'-biquinazoline (7), 2,2'biquinoxaline (8), 2,2'-bibenzoxazole (10) and 2,2-bibenzothiazole (11) all exist in the solid state in centrosymmetric, planar conformations that minimise their dipole moments and maximise both conjugation between the rings and various types of attractive intermolecular associations. In contrast, 4,4'-biquinazoline (9) and 1,1'-bibenzotriazole (12) display non-planar conformations due to repulsive intramolecular interactions.

\section{Introduction}

Aromatic biheterocycles represent an important class of compounds with relevance in many areas of chemistry. ${ }^{1}$ For example, they exist as subunits in many naturally occurring compounds and act as models for higher oligo- and polyheterocycles. ${ }^{2}$ By far their most common use, however, is as chelating (e.g. 2,2'-bipyridine) or bridging (e.g. 4,4'-bipyridine) ligands for metal coordination. ${ }^{3}$ Such metal complexes have numerous applications in inorganic, organometallic and analytical chemistry. ${ }^{4}$ The syntheses, structures and properties of all the parent aromatic biheterocycles have been comprehensively reviewed. ${ }^{1}$

The conformations of biheterocycles have been the focus of much experimental and computational study. ${ }^{1}$ From such investigations it has been proposed that the conformation about the inter-ring bond in biheterocycles is determined by the nature of the adjacent atom groupings. ${ }^{5}$ Specifically, two types of interactions have been identified as destabilising [viz $\mathrm{C}-\mathrm{H}^{\cdots} \mathrm{H}-\mathrm{C}$ (1a) and $\mathrm{N}: \cdots: \mathrm{N}(\mathbf{1 b})]$ and one as stabilising $\left[\mathrm{C}-\mathrm{H}^{\cdots}: \mathrm{N}(\mathbf{1 c})\right]$. Such arguments have been used to rationalise the s-trans conformations of molecules such as 2,2'-bipyridine (2) and 1,1'-

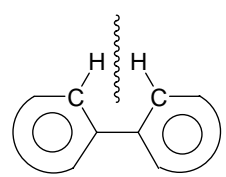

$1 \mathrm{a}$

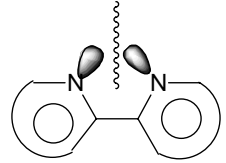

1b

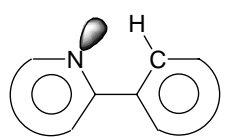

$1 c$

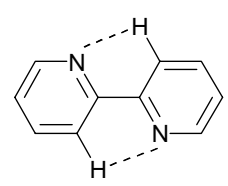

2

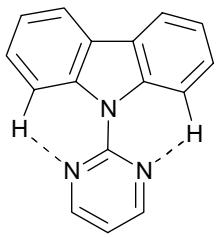

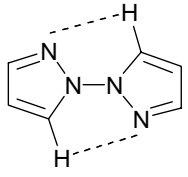

3

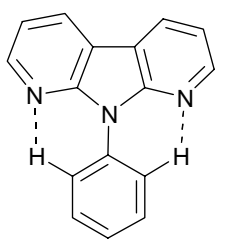

5 bipyrazole (3). ${ }^{5}$ Considerable computational and experimental evidence supports the importance of these factors in the gas phase and in solution. However, we believe that these factors are likely to be much less important in the solid state. Whilst both intermolecular and intramolecular $\mathrm{C}-\mathrm{H}^{\cdots}: \mathrm{N}$ interactions are well known to influence solid state crystal structures, these are energetically rather weak interactions. ${ }^{6}$ We contend that factors such as molecular packing, conjugation issues and dipole minimisation effects are much more influential in controlling the solid state conformations of such molecules. ${ }^{7}$ For example, biphenyl is well known to be non-planar in both the gas phase and in solution, but is strictly planar in the crystalline state, ${ }^{8}$ despite the presence of two repulsive interactions of type (1a). Similarly, 2,2'-bipyrimidine is planar, ${ }^{9}$ but possesses only interactions of type (1b).

Nevertheless, the conformations of biheterocycles and higher oligomers in the solid state are regularly rationalised on the basis of the above arguments. In an elegant study some years ago, ${ }^{10}$ Avendano et al. reported the syntheses and X-ray crystal structures of a family of aza-substituted 9-phenylcarbazoles as a test for the validity of these proposals. They interpreted the observation that the pyrimidyl derivative (4) was close to planar (torsional angle $7.4^{\circ}$ ) as strong support for the existence of intramolecular $\mathrm{C}(\mathrm{Ar})-\mathrm{H}^{\cdots}: \mathrm{N}$ hydrogen bonds, despite the fact that the isomeric compound (5) was far from planar.

In the case of symmetrical biheterocycles the situation can become more complex, since by adopting an s-trans coplanar conformation about the inter-ring bond, and only by doing so, the dipole moment of the molecule reduces to zero. We believe that this is a very important factor in determining the solid state conformations of such molecules. Indeed, by adopting an s-trans coplanar conformation the molecule not only minimises its dipole moment but also facilitates crystallisation about a crystallographic centre of inversion. This in turn allows crystallisation in a non-polar, centrosymmetric space group. Furthermore, a planar conformation allows for more efficient $\pi$ $\pi$ stacking interactions between molecules, such as one of the various herringbone packing motifs common for planar aromatic molecules which crystallise in $\mathrm{P} 2_{1} / \mathrm{c}$, the most common of all space groups. ${ }^{11}$

In order to test the hypothesis that this factor is indeed the most important in determining solid state conformations, we herein describe the accurate, low temperature X-ray crystal structures of seven symmetrical aromatic biheterocycles. Since each of these compounds is also capable of acting as a chelating and/or bridging ligand, this study is also likely to be of interest to chemists studying the coordination and supramolecular chemistry of such ligands. 


\section{Results and discussion}

The seven biheterocycles studied (6-12) were prepared by literature procedures and chosen for their ability to test the factors discussed above. Each could potentially exist in two planar conformations of different symmetry. Accurate X-ray crystal structure determinations of all seven compounds were performed at low temperature. 3,3'-Biquinoline (6), 2,2'biquinazoline (7) and 2,2'-biquinoxaline (8) are all aza-2,2'binaphthyls and were all found to exist in a planar conformation in the solid state (Fig. 1-3). Furthermore, each exists with an strans conformation about the inter-ring bond and crystallises about a crystallographic centre of inversion with only half a molecule in the asymmetric unit.
6<smiles>c1ccc2nc(-c3cnc4ccccc4n3)cnc2c1</smiles>

8<smiles>c1ccc2oc(-c3nc4ccccc4o3)nc2c1</smiles>

10<smiles>c1ccc2sc(-c3nc4ccccc4s3)nc2c1</smiles>

11

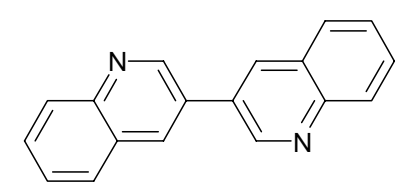

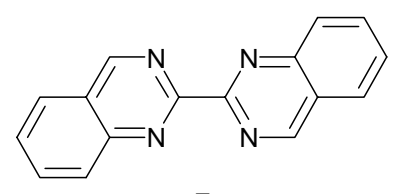

7

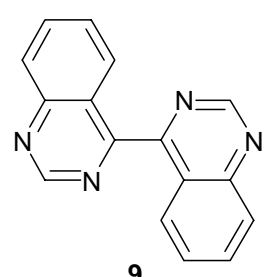

9

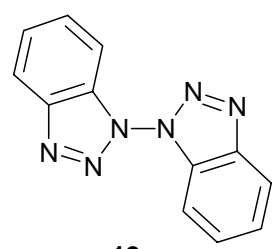

12
Both possible planar conformations of the biquinoline (6) have two interactions of type 1a and this compound would therefore be expected to be non-planar. Similarly, the biquinazoline (7) can only have interactions of type 1b in a planar form and again would be expected to be non-planar. In contrast the biquinoxaline (8) could have two very different planar conformations, one with only interactions of type 1c (as is found to be the case) and another with one type 1a and one type 1b interaction. The fact that all three compounds are found to exist in planar centrosymmetric conformations in the solid state supports our contention these these types of interactions are not the most important in determining the solid state conformation. We believe that these structures are favoured as they represent the only conformations with no dipole moments and that the resulting planar conformations maximise energetically favourable intermolecular forces.

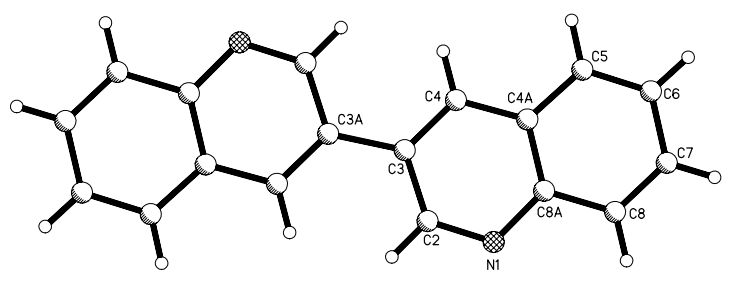

Fig. 1 Perspective view of $\mathbf{6}$, with atomic labelling shown. Selected bond lengths $(\AA)$ and angles $\left({ }^{\circ}\right)$ : N1-C2 1.303(3), N1-C8A 1.356(3), C2-C3 1.404(3), C3-C4 1.351(3), C3-C3A 1.473(4), C4-C4A 1.391(3), C2-N1C8A 117.1(2), N1-C2-C3 125.3(2), C4-C3-C2 116.6(2), C4-C3-C3A 123.5(3), C2-C3-C3A 119.9(3), C3-C4-C4A 120.3(2), N1-C8A-C4A 122.0(2), N1-C8A-C8 118.1(2).

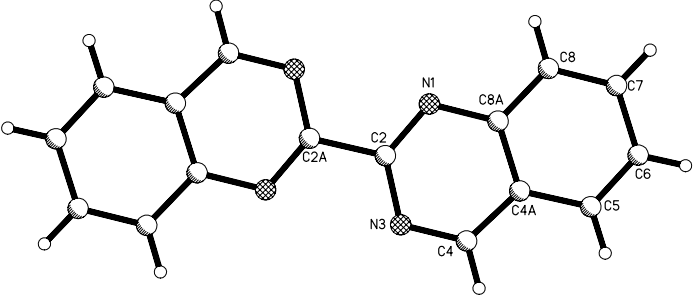

Fig. 2 Perspective view of 7, with atomic labelling shown. Selected bond lengths $(\AA)$ and angles $\left({ }^{\circ}\right)$ : N1-C2 1.318(3), N1-C8A 1.371(3), C2-N3 1.379(2), C2-C2A 1.507(4), N3-C4 1.312(3), C2-N1-C8A 116.29(2), N1-C2-N3 127.2(2), N1-C2-C2A 117.4(2), N3-C2-C2A 115.4(2), C4N3-C2 116.4(2), N3-C4-C4A 122.8(2), N1-C8A-C4A 121.3(2).

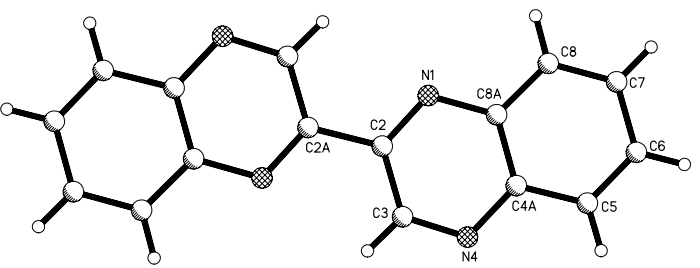

Fig. 3 Perspective view of $\mathbf{8}$, with atomic labelling shown. Selected bond lengths $(\AA)$ and angles $\left({ }^{\circ}\right)$ : N1-C2 1.318(1), N1-C8A 1.370(1), C2-C3 1.421(2), C2-C2A 1.478(2), C3-N4 1.313(1), N4-C4A 1.368(1), C2-N1C8A 116.6(1), N1-C2-C3 121.6(1), N1-C2-C2A 118.2(1), C3-C2-C2A 120.3(1), N4-C3-C2 123.5(1), C3-N4-C4A 115.96(9), N4-C4A-C5 119.5(1), N4-C4A-C8A 121.1(1), N1-C8A-C8 119.7(1), N1-C8A-C4A 121.20(9).

This is supported by inspection of the molecular packing which is very similar for all three compounds. Fig. 4 shows a packing diagram for compound 6. The molecules pack in a $\gamma$ herringbone manner ${ }^{11}$ with the molecules packed along the $b$ axis of the unit cell. The molecules arrange with each quinoline ring stacked on top of another, but offset so that the linking carbon atom lies over the centre of an adjacent benzo-fused ring. It has been proposed that such offset stacking maximises the electrostatic attraction between coplanar aromatic systems. ${ }^{12}$ The distances between the meanplanes in the structures of $\mathbf{6 , 7}$ and 8 are 3.398(5), 3.410(4) and 3.428(3) $\AA$, respectively, which are similar to the distance between the planes of carbon atoms in graphite $(3.35 \AA)$. The $\gamma$-herringbone pattern also allows edge-toface interactions to occur between molecules of adjacent stacks. These $\mathrm{C}-\mathrm{H}^{\cdots} \pi$ interactions lie in the range $2.7-2.9(5) \AA$, which are typical values for these types of interactions. ${ }^{13}$ The angles between the molecules of adjacent stacks varies from $64^{\circ}$, in the structures of $\mathbf{6}$ and $\mathbf{7}$, to $80^{\circ}$ for $\mathbf{8}$.

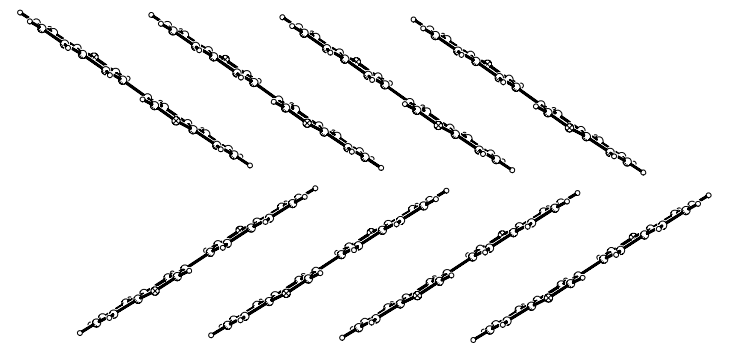

Fig. 4 View of the packing of 6, showing $\pi-\pi$ stacking and edge-to-face interactions. 
In contrast to the planar structures found for compounds $\mathbf{6 - 8}$, 4,4'-biquinazoline (9) is non-planar in the solid state (Fig. 5). This is in direct conflict with the predictions discussed in the Introduction, since a centrosymmetric planar conformation would facilitate two $\mathrm{C}-\mathrm{H}^{\cdots}: \mathrm{N}$ interactions, similar to those depicted in the structure of 4 . This aza-1,1-binaphthyl again crystallises with only half a molecule in the asymmetric unit, but this time about a two-fold rotation axis. The angle between the meanplanes of the quinazoline rings is $50.9(1)^{\mathrm{o}}$. $^{14}$

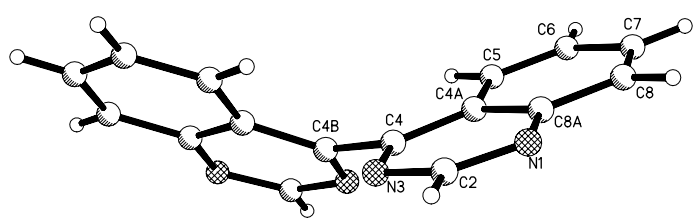

Fig. 5 Perspective view of $\mathbf{9}$, with atomic labelling shown. Selected bond lengths $(\AA)$ and angles $\left(^{\circ}\right)$ : N1-C2 1.318(4), N1-C8A 1.377(4), C2-N3 $1.362(4), \mathrm{N} 3-\mathrm{C} 41.322(4), \mathrm{C} 4-\mathrm{C} 4 \mathrm{~A} 1.436(4), \mathrm{C} 4-\mathrm{C} 4 \mathrm{~B} 1.503(6), \mathrm{C} 2-\mathrm{N} 1-$ C8A 116.1(3), N1-C2-N3 127.7(3), C4-N3-C2 117.2(3), N3-C4-C4A 121.7(3), N3-C4-C4B 116.1(3), C4A-C4-C4B 122.2(3), N1-C8A-C4A 121.3(3).

Once again, there is $\pi-\pi$ stacking between adjacent molecules with the meanplanes of the quinazoline rings separated by $3.531(5) \AA$, indicated a slightly weaker interaction than for the planar molecules described above. Also, there are no edge-to-face $\mathrm{C}-\mathrm{H}^{\cdots} \pi$ intermolecular interactions between adjacent chains of molecules in this case. We believe that the centrosymmetric planar structure 9a is actually destabilised by the $\mathrm{C}-\mathrm{H}^{\cdots}: \mathrm{N}$ interaction shown, as this arrangement of an aza1,1'-binaphthyl brings these atoms closer than the energy minimum for such an interaction. Support for this proposal comes from the observation that an analogous, complementary interaction exists in $8,8^{\prime}$-biquinoline (13), which is also nonplanar in the solid state $\left(\right.$ angle between planes $\left.=98.6^{\circ}\right){ }^{15}$

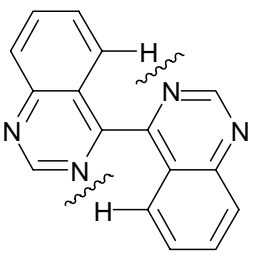

$9 a$

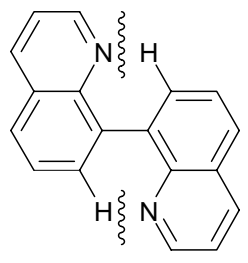

13
We next turned our attention to the bibenzazoles (10-12). 2,2'-Bibenzoxazole (10) and 2,2-bibenzothiazole (11) both crystallise in a planar conformation about a crystallographic centre of inversion (Fig. 6,7). During the course of this study the room temperature X-ray structure of $\mathbf{1 0}$ was reported by other

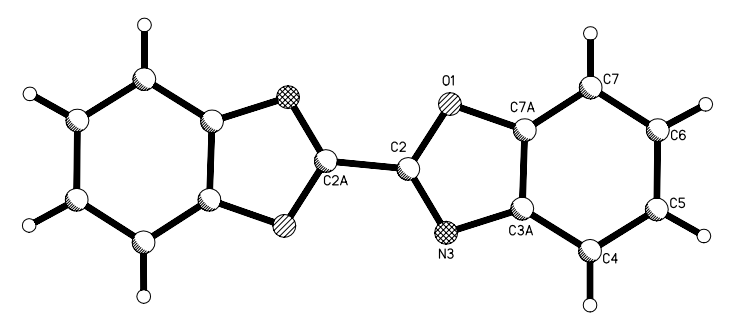

Fig. 6 Perspective view, with atom labelling, of 10. Selected bond lengths $(\AA)$ and angles $\left(^{\circ}\right)$ : O1-C7A 1.388(2), C2-N3 1.301(2), N3-C3A 1.396(3), C3A-C7A 1.378(3), C5-C6 1.391(3), C7A-C7 1.373(3), O1-C2 1.352(3), C2-C2' 1.449(4), C3A-C4 1.399(3), C4-C5 1.378(3), C6-C7 1.391(3), C7A-O1-C2 103.4(2), N3-C2-O1 116.7(2), C2-N3-C3A 103.0(2), C3A-C7A-O1 107.3(2).

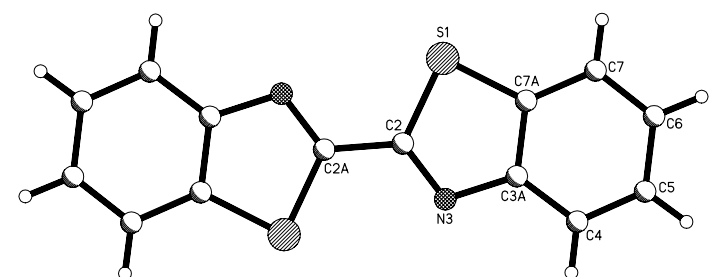

Fig. 7 Perspective view, with atom labelling, of 11. Selected bond lengths $(\AA)$ and angles $\left({ }^{\circ}\right)$ :S1-C2 1.741(2), S1-C7A 1.730(2), C2-N3 $1.300(3), \mathrm{C} 2-\mathrm{C} 2$ ' 1.459(4), N3-C3A 1.390(2), C3A-C7A 1.408(3), C5C6 1.401(3), C7-C7A 1.400(3), C3A-C4 1.397(3), C4-C5 1.375(3), C6C7 1.378(3), C7A-S1-C2 88.12(9), N3-C2-S1 117.6(2), C2-N3-C3A 109.(2), C3A-C7A-S1 110.0(1).

workers, ${ }^{16}$ but is included here for strict comparison under identical conditions to the other compounds. Once again, these centrosymmetric planar conformations maximise conjugation between the ring systems, minimise the dipole moments and facilitate the strongest intermolecular stacking interactions. Both compounds pack in a $\gamma$-herringbone manner with $\pi-\pi$ stacking of adjacent molecules separated by 3.386(4) and 3.620(5) $\AA$ for 10 and 11 , respectively. There are also edge-to-face $\mathrm{C}-\mathrm{H}^{\cdots} \pi$ interactions $(<3.0 \AA)$ between adjacent stacks of molecules.

1,1'-Bibenzotriazole (12) crystallises in the chiral orthorhombic space group $\mathrm{P} 2{ }_{1} 2_{1} 2_{1}$, with the asymmetric unit containing one full molecule. Both rings of the ligand are planar [mean deviation from the plane $=0.005(1) \AA$ for both rings], and are very nearly orthogonal to each other $\left[84.5(1)^{\circ}\right]$ (Fig. 8). This non-planar conformation is not expected on the basis of the types of interaction discussed in the Introduction as a centrosymmetric planar conformation would have two C-H...N interactions similar to those found in compound 4. As before, we believe that fusion of the benzo-rings adjacent to the inter-ring bond disfavours a planar conformation.

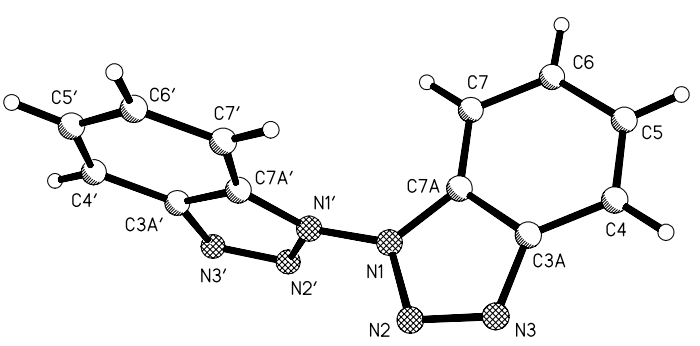

Fig. 8 Perspective view, with atom labelling of the contents of the asymmetric unit, of 12. Selected bond lengths $(\AA)$ and angles $\left({ }^{\circ}\right)$ : N1-N2 1.366(5), N2-N3 1.304(5), C3A-C7A 1.398(6), C7A-N1 1.377(5), N1N1' 1.366(5), N1'-N2' 1.391(5), N2'-N3' 1.294(5), C3A'- C7A' 1.387(6), C7A'-N1' 1.379(5), N1-N2-N3 106.9(3), N2-N1-C7A 128.0(4), N1'-N2'N3' 107.6(3).

The molecular packing of $\mathbf{1 2}$ is intriguing and involves a complex system of intermolecular interactions. As shown in Fig. 9, significant interactions connect adjacent molecules related by all three orthogonal screw axes. Molecules related by the twofold screw axis in the $a$-direction exhibit head-to-tail $\pi-\pi$ stacking with a separation of $c a 3.41 \AA$. Two sets of interactions connect molecules along the $b$-axis; the first is a face-to-face stacking interaction between molecules related by a screw axis and the second is a $\mathrm{C}-\mathrm{H}^{\cdots} \mathrm{N}(2.56 \AA)$ interaction between molecules in adjacent units cells. Finally, the above chains of molecules are connected along the $c$-axis by interdigitation of the $\pi-\pi$ stacking. The combined effects of these numerous intermolecular interactions are almost certainly sufficient to override the weaker intramolecular interactions discussed above. 


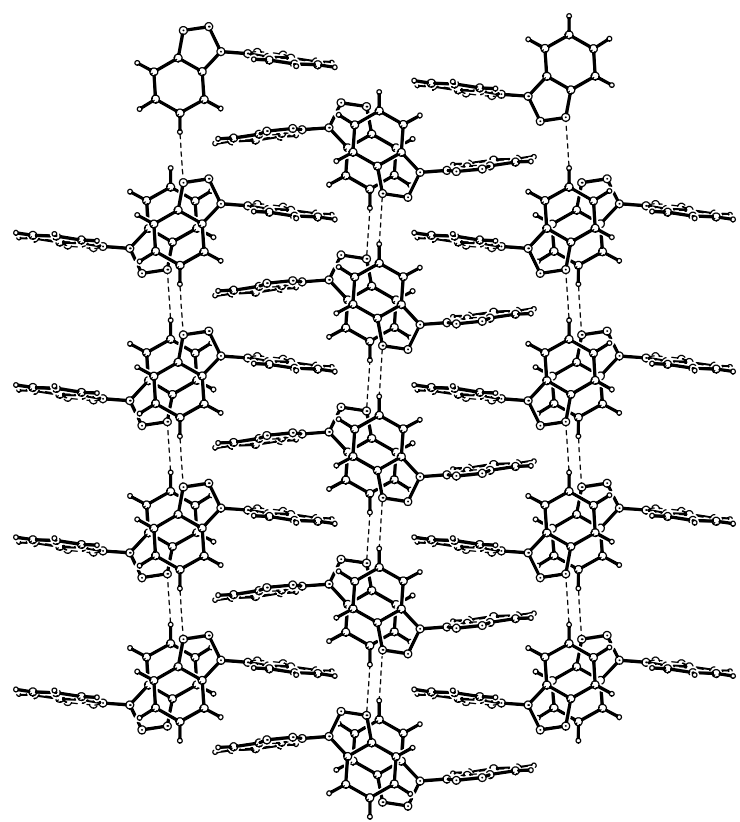

Fig. 9 View of the molecular packing down the $a$-axis in the crystal structure of 12 .

\section{Conclusions}

The X-ray crystal structures of the seven symmetrical biheterocycles 6-12 support our contention that the solid state conformations of such compounds are dominated by a proclivity to adopt a planar centrosymmetric structure. This serves to maximise conjugation between the rings, minimise the dipole moment and maximise favourable intermolecular interactions and is sufficient to override other (weaker) intramolecular interactions. However, for molecules containing benzo-fused rings adjacent to the inter-ring bond repulsive interactions exist that lead to non-planar conformations.

\section{Experimental}

\section{Sample preparations}

3,3'-Biquinoline (6) was synthesised by the homocoupling of 3bromoquinoline, following the procedure of Hassan et al., ${ }^{17}$ and recrystallised from methanol. 2,2'-Biquinazoline (7) was prepared by the ring closing reaction of ammonia with $\mathrm{N}, \mathrm{N}^{\prime}$-(di$o$-formylphenyl)oxanilide, following the method of Armarego and Willette, ${ }^{18}$ which directly furnished crystals suitable for structure determination. 2,2'-Biquinoxaline (8) was synthesised using the method of Chupakhin et al., ${ }^{19}$ and was crystallised by diffusion of methanol into a dichloromethane solution of the compound. 4,4'-Biquinazoline (9) was synthesised using the method of Armarego and Willette, ${ }^{18}$ by reaction of quinazoline with sodium cyanide, followed by oxidation of the intermediate with manganese dioxide. Crystals suitable for X-ray structure determination were obtained by slow evaporation of an acetonitrile solution of the compound. 2,2'-Bibenzoxazole (10) and 2,2-bibenzothiazole (11) were prepared by polyphosphoric acid-induced cyclocondensation reactions of diethyl oxalate with 2-aminophenol and 2-aminothiophenol, respectively, according to literature procedures. ${ }^{20,21} \mathrm{X}$-ray quality crystals were obtained by recrystallisation from chloroform and dioxane, respectively. 1,1'-Bibenzotriazole (12) was obtained from 2,2'diaminoazobenzene by diazotisation followed by reductive cyclisation, as described by Harder et al.,2 and was recrystallised from ethyl acetate.

\section{X-Ray Crystallography}

The crystal data, data collection and refinement parameters are given in Table 1. Measurements were made with a Bruker CCD area detector or a Siemens P4s four-circle diffractometer using graphite monochromatised Mo $\mathrm{K} \alpha(\lambda=0.71073 \AA)$ radiation. The intensities were corrected for Lorentz and polarisation effects and for absorption. The structures were solved by direct methods using SHELXS, ${ }^{23}$ and refined on $F^{2}$ using all data by full-matrix least-squares procedures using SHELXL-97. ${ }^{24}$ All non-hydrogen atoms were refined with anisotropic displacement parameters. Hydrogen atoms were included in calculated positions with isotopic displacement parameters 1.2 times the isotropic equivalent of their carrier carbon atoms. The functions

Table 1 Crystal data and X-ray experimental data for compounds 6-12

\begin{tabular}{|c|c|c|c|c|c|c|c|}
\hline Compound & 6 & 7 & 8 & 9 & 10 & 11 & 12 \\
\hline Collection device & $\mathrm{CCD}$ & CCD & $\mathrm{CCD}$ & $\mathrm{CCD}$ & $\mathrm{P} 4 \mathrm{~s}$ & $\mathrm{P} 4 \mathrm{~s}$ & $\mathrm{CCD}$ \\
\hline Empirical formula & $\mathrm{C}_{18} \mathrm{H}_{12} \mathrm{~N}_{2}$ & $\mathrm{C}_{16} \mathrm{H}_{10} \mathrm{~N}_{4}$ & $\mathrm{C}_{16} \mathrm{H}_{10} \mathrm{~N}_{4}$ & $\mathrm{C}_{16} \mathrm{H}_{10} \mathrm{~N}_{4}$ & $\mathrm{C}_{14} \mathrm{H}_{8} \mathrm{~N}_{2} \mathrm{O}_{2}$ & $\mathrm{C}_{14} \mathrm{H}_{8} \mathrm{~N}_{2} \mathrm{~S}_{2}$ & $\mathrm{C}_{12} \mathrm{H}_{8} \mathrm{~N}_{6}$ \\
\hline Formula weight & 256.30 & 258.28 & 258.28 & 258.28 & 236.22 & 268.346 & 236.24 \\
\hline Temperature (K) & $163(2)$ & $170(2)$ & $163(2)$ & $163(2)$ & $163(2)$ & $163(2)$ & $168(2)$ \\
\hline Crystal system & Monoclinic & Monoclinic & Monoclinic & Monoclinic & Monoclinic & Monoclinic & Orthorhombic \\
\hline Space group & $\mathrm{P} 2{ }_{1} / \mathrm{n}$ & $\mathrm{P} 2{ }_{1} / \mathrm{n}$ & $\mathrm{P} 2_{1} / \mathrm{n}$ & $\mathrm{C} 2$ & $\mathrm{P} 2{ }_{1} / \mathrm{n}$ & $\mathrm{P} 2{ }_{1} / \mathrm{c}$ & $\mathrm{P} 2{ }_{1} 2_{1} 2_{1}$ \\
\hline Cell dimensions: a $(\AA)$ & $8.964(6)$ & $8.557(4)$ & $5.779(3)$ & $22.523(17)$ & $4.627(1)$ & $8.993(1)$ & $7.277(3)$ \\
\hline $\mathrm{b}(\AA)$ & $6.265(4)$ & $6.330(3)$ & $5.350(3)$ & $3.912(3)$ & $16.480(2)$ & $5.998(1)$ & $7.639(3)$ \\
\hline c $(\AA)$ & $11.241(8)$ & $11.841(5)$ & $18.906(10)$ & $6.979(5)$ & $7.004(1)$ & $11.445(2)$ & $19.940(8)$ \\
\hline$\beta\left(^{\circ}\right)$ & $107.109(18)$ & $106.689(6)$ & $91.466(7)$ & $106.140(10)$ & $94.34(1)$ & $110.87(1)$ & 90 \\
\hline Volume $\left(\AA^{3}\right)$ & $603.3(7)$ & $614.4(5)$ & $584.3(5)$ & $590.6(7)$ & $535.5(2)$ & $575.9(2)$ & $1108.5(7)$ \\
\hline Z & 2 & 2 & 2 & 2 & 2 & 2 & 4 \\
\hline Density (calc.) $\left(\mathrm{Mg} / \mathrm{m}^{3}\right)$ & 1.411 & 1.396 & 1.468 & 1.452 & 1.473 & 1.548 & 1.416 \\
\hline Abs. coefficient $\left(\mathrm{mm}^{-1}\right)$ & 0.084 & 0.087 & 0.092 & 0.091 & 0.101 & 0.441 & 0.094 \\
\hline $\mathrm{F}(000)$ & 268 & 268 & 268 & 268 & 244 & 276 & 488 \\
\hline Crystal size $(\mu \mathrm{m})$ & $350 \times 250 \times 10$ & $750 \times 250 \times 120$ & $300 \times 300 \times 150$ & $590 \times 200 \times 50$ & $340 \times 220 \times 180$ & $770 \times 210 \times 130$ & $510 \times 250 \times 160$ \\
\hline Theta range $\left({ }^{\circ}\right)$ & $2.57-25.05$ & $2.61-26.46$ & $2.16-26.36$ & $3.04-25.20$ & $2.47-26.00$ & $2.42-26.99$ & $2.86-25.05$ \\
\hline Reflections collected & 6814 & 7439 & 6979 & 3477 & 1733 & 1618 & 13016 \\
\hline Independent data $[\mathrm{R}(\mathrm{int})]$ & $1070[0.068]$ & $1253[0.030]$ & $1190[0.013]$ & $619[0.082]$ & $1044[0.035]$ & $1261[0.027]$ & $1170[0.098]$ \\
\hline Observed data $[\mathrm{I}>2 \sigma(\mathrm{I})]$ & 532 & 891 & 954 & 466 & 741 & 964 & 929 \\
\hline Data / parameters & $1070 / 92$ & $1253 / 91$ & $1190 / 91$ & $619 / 92$ & $1044 / 82$ & $1261 / 82$ & $1170 / 163$ \\
\hline Goodness-of-fit on $\mathrm{F}^{2}$ & 0.843 & 1.026 & 1.064 & 0.965 & 0.920 & 0.928 & 1.146 \\
\hline $\mathrm{wR}_{2}$ (all data) & 0.1043 & 0.1328 & 0.1112 & 0.1082 & 0.1126 & 0.0876 & 0.1331 \\
\hline $\mathrm{R} 1[\mathrm{I}>2 \sigma(\mathrm{I})]$ & 0.0433 & 0.0547 & 0.0359 & 0.0457 & 0.0441 & 0.0342 & 0.0558 \\
\hline
\end{tabular}


minimised were $\Sigma w\left(F_{\mathrm{o}}^{2}-F_{\mathrm{c}}^{2}\right)$, with $w=\left[\sigma^{2}\left(F_{\mathrm{o}}^{2}\right)+\mathrm{a} P^{2}+\mathrm{b} P\right]^{-1}$, where $P=\left[\max \left(F_{\mathrm{o}}\right)^{2}+2 F_{\mathrm{c}}^{2}\right] / 3$. The bonding geometries within the individual rings of the various compounds were all found to be similar to those observed in structurally related compounds containing the same ring systems. ${ }^{25}$

Crystallographic data, as CIF files, have been deposited with the Cambridge Crystallographic Data Centre (CCDC No 253922 - 253928). Copies can be obtained free of charge from: The Director, CCDC, 12 Union Road, Cambridge CB2 1EZ, U.K. (email: deposit@ccdc.cam.ac.uk).

\section{References}

1 P. J. Steel, Adv. Heterocycl. Chem., 1997, 67, 1 and references therein.

2 G. R. Newkome, A. K. Patri, E. Holder and U. S. Schubert, Eur. J. Org. Chem., 2004, 235.

3 C. Kaes, A. Katz and M. W. Hosseini, Chem. Rev., 2000, 100, 3553.

4 P. J. Steel, Molecules, 2004, 9, 440 and references therein.

5 M. L. Castellanos, S. Olivella, N. Roca, J. de Mendoza and J. Elguero, Can. J. Chem., 1984, 62, 687; S. P. Singh, D. Kumar, D. Kumar, A. Martinez and J. Elguero, J. Heterocycl. Chem., 1996, 33, 323.

6 G. R. Desiraju, Acc. Chem. Res., 2002, 35, 565; A. Nangia, CrystEngComm, 2002, 4, 93; M. Mazik, D. Blaser and R. Boese, Tetrahedron Lett., 2000, 41, 5827; V. R. Thalladi, A. Gehrke and R. Boese, New. J. Chem., 2000, 24, 463; J. J. M. Amoore, L. R. Hanton and M. D. Spicer, Dalton Trans., 2003, 1056 and references therein.

7 J. K. Whitesell, R. F. Davis, L. L. Saunders, R. J. Wilson and J. P. Feagins, J. Am. Chem. Soc., 1991, 113, 3267.

8 G. B. Robertson, Nature (London), 1961, 191, 593.

9 L. Fernholt, C. Romming and S. Samdal, Acta Chem. Scand., 1981, 35A, 707; D. J. Berg, J. M. Boncella and R. A. Anderson, Organometallics, 2002, 21, 4622.

10 C. Avendano, M. Espada, B. Ocana, S. Garcia-Granda, M. del Rosario Diaz, B. Tejerina, F. Gomez-Beltran, A. Martinez, and J. Elguero, J. Chem. Soc., Perkin Trans. 2, 1993, 1547.

11 G. R. Desiraju and A. Gavezzotti, Acta Crystallogr., Sect. B, 1989, 45, 473 .

12 C. A. Hunter, K. R. Lawson, J. Perkins, and C. J. Urch, J. Chem. Soc., Perkin Trans. 2, 2001, 651; C. Janiak, J. Chem. Soc., Dalton Trans., 2000, 3885 and references therein.

13 C. A. Hunter and J. K. M. Sanders, J. Am. Chem. Soc., 1990, 112 , 5525; T. Steiner, Angew. Chem. Int. Ed., 2002, 41, 48; M. Nishio, CrystEngComm, 2004, 6, 130; E. A. Meyer, R. K. Castellano and F. Diederich, Angew. Chem. Int. Ed., 2003, 42, 1210; W. B. Jennings, B. M. Farrell and J. F. Malone, Acc. Chem. Res., 2001, 34, 885.

14 The crystal structure of a ruthenium(II) complex of 9 has recently been reported. L. Ademi, E. C. Constable, C. E. Housecroft, M. Neunurger and S. Schaffner, Dalton Trans., 2003, 4565.

15 M. Lenner and O. Lindgren, Acta Crystallogr., Sect. B, 1976, 32, 1903.

16 F. E. Hahn, L. Imhof and T. Lügger, Acta Crystallogr., Sect. C, 1998, 54, 668

17 J. Hassan, V. Penalva, L. Lavenot, C. Gozzi, and M. Lemaire, Tetrahedron, 1998, 54, 13793.

18 W. L. F. Armarego and R. E. Willette, J. Chem. Soc., 1965, 1258.

19 O. N. Chupakhin, E. O. Sidorov, S. M. Shein, and I. I. Bil'kis, Zh. Org. Khim., 1976, 12, 2464.

20 F. Kehrmann and C. Bener, Helv. Chim. Acta, 1925, 8, 16.

21 C. Rai and J. B. Braunworth, J. Org. Chem., 1961, 26, 3434.

22 R. J. Harder, R. A. Carboni and J. E. Castle, J. Am. Chem. Soc., $1967, \mathbf{8 9}, 2643$.

23 G. M. Sheldrick, Acta Crystallogr., Sect. A, 1990, 46, 467.

24 G. M. Sheldrick, SHELXL-97, University of Göttingen, Germany, 1997.

25 F. H. Allen and W. D. S. Motherwell, Acta Crystallogr., Sect. B, 2002, 58, 407; F. H. Allen and R. Taylor, Chem. Soc. Rev., 2004, 33, 463. 
